\title{
The first hundred years
}

\section{The Bohr atom is unquestionably a landmark in the history of physics. A century after its publication, it has inspired a remarkably diverse and ever-growing field of research.}

Between July and November 1913, Niels Bohr published a seminal trilogy of papers ${ }^{1}$ in the Philosophical Magazine. In them, he put forward a new model for the hydrogen atom, combining Ernest Rutherford's idea of a positively charged nucleus surrounded by electrons with Max Planck's concept of radiation quanta. The model was (as Daniel Kleppner has commented in a review ${ }^{2}$ ) "remarkable for its daring introduction of radical ideas and its cavalier disregard of classical electromagnetic theory".

To mark the centenary of the 'Bohr atom', several authors have revisited the historical context of his ideas and the subsequent refinements of his theory of atomic structure (see, for example, refs 3,4). Furthermore, the strong influence of Bohr's family, particularly his wife Margrethe, on his work has been brought to light in recently published correspondence (Love, Literature, and the Quantum Atom is reviewed on page 602).

But to fully grasp the significance of Bohr's atomic model in the history of physics - and of modern science in general - one should look at its legacy, in particular the spectacular developments that have taken place in the field of atomic physics in the last hundred years. Just a few of those major experimental landmarks are indicated in the timeline here (loosely based on ref. 2), which also records the Nobel prizes awarded to some of the leading figures in the field for their contributions to atomic physics.

In fact, although it can be traced back to the time of Bohr and Rutherford, the field of atomic physics only really became recognizable as a separate discipline in the 1940s. Bohr's 1913 model was "fundamentally incomplete" ${ }^{\text {: its completion }}$ required further scientific progress; for example, the development of quantum mechanics and discoveries such as that of the neutron in 1932. Twenty years after the original papers, a more definitive theory of atomic structure had been finalized.

On the practical side, by the 1930 s Isidor Rabi had already started doing molecular-beam experiments and had established the basis of nuclear magnetic resonance. In the decade that followed, Willis Lamb elucidated the fine structure of hydrogen, which led to the development of quantum electrodynamics. The 1950 s saw

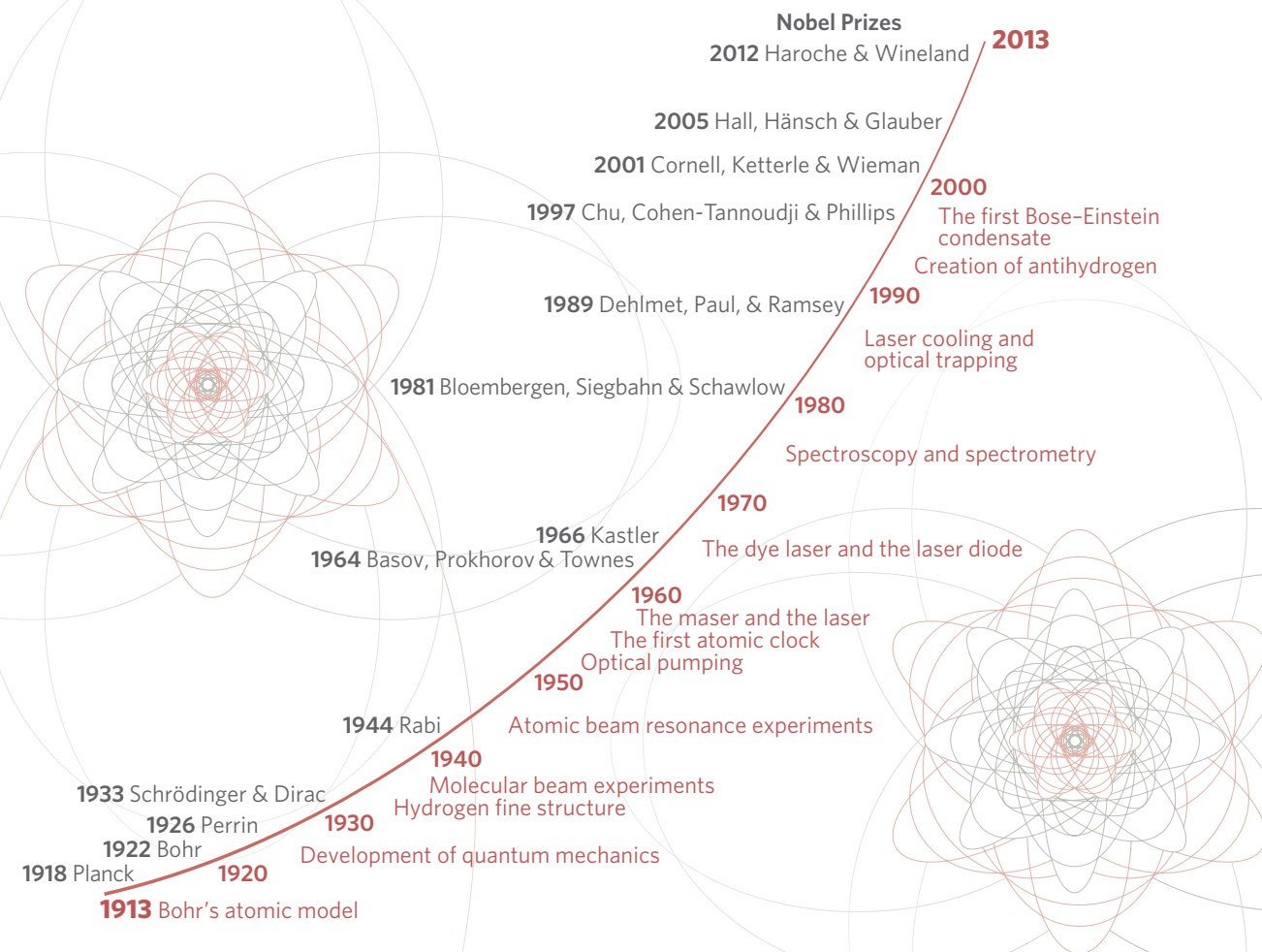

the discovery of optical pumping and the invention of the maser and the laser - a turning point in atomic physics, enabling a whole new generation of experiments. Moreover, the subsequent development of the dye laser and the laser diode made even more experimentally possible.

Many applications that we take for granted today have their roots in atomic physics: nuclear magnetic resonance, as already mentioned; spectroscopy and spectrometry; and the global positioning system, made possible by atomic clocks. Some predate the advent of the laser, some were only achievable or improved after that point. Recent progress has been made and great opportunities for future progress lie - in fields such as atomic magnetometry, and in quantum technologies including quantum information processors and quantum memories.

In the 1980s came the laser cooling and optical trapping that made the coherent control of atomic systems possible - and so began a new area of research using ultracold atomic gases. This field has become progressively intertwined with condensedmatter physics, bringing fresh insight into long-standing problems: for instance, the observation $^{5}$ of the phase transition from superfluid to Mott insulator using ultracold atoms, made in 2002. Now, as evidenced in this issue, cold-atom experimenters are even tackling problems in such disparate fields as cosmology (the spontaneous creation of Kibble-Zurek solitons in Bose-Einstein condensates reported on page 656; accompanied by a News \& Views article on page 605); thermodynamics (the emergence of thermal correlations in an isolated quantum many-body system, page 640; with an associated News \& Views on page 607); and even statistical physics - as recorded in the Progress Article by Eric Lutz and Ferruccio Renzoni (page 615).

As physicists gather ${ }^{6}$ this month in Copenhagen to celebrate Bohr's great legacy, it is clear that more exciting advances can be years, but in the future close at hand.

\section{References}

1. Bohr, N. Philos. Mag. 26, 1-25; 476-502; 857-875 (1913).

2. Kleppner, D. Rev. Mod. Phys. 71, S78-S84 (1999)

3. Kragh, H. Phys. Today 66, 36-41 (May 2013).

4. www.nature.com/bohr100

5. Greiner, M. et al. Nature 415, 39-44 (2002).

6. http://bohr2013.nbi.ku.dk/english anticipated - not even in the next hundred 